\title{
Expectativas de migración internacional en estudiantes de enfermería en México, Distrito Federal
}

\author{
Yetzi Rosales-Martínez, M en CS, (I) Gustavo Nigenda, PhD, (I) Omar Galárraga, PhD, (I) \\ José Arturo Ruiz-Larios, Lic en Soc. (I)
}

Rosales-Martínez Y, Nigenda G, Galárraga O, Ruiz-Larios JA. Expectativas de migración internacional en estudiantes de enfermería en México, Distrito Federal. Salud Publica Mex 2010;52:244-253.

\section{Resumen}

Objetivo.Analizar los factores asociados con la expectativa de migrar al extranjero en estudiantes de licenciatura en enfermería de escuelas públicas en México, Distrito Federal. Material y métodos. Se realizó un estudio transversal con una muestra no probabilística de 420 estudiantes. Se construyó un modelo logístico multivariado. Resultados. El $69 \%$ de los informantes expresó la intención de migrar para trabajar (65\%) o estudiar (26\%). El 50\% elegiría como destino Canadá, seguido de España y Estados Unidos. Las variables asociadas con la expectativa de migrar fueron: edad, ingreso, tener familiares en el extranjero y percibir malas condiciones laborales/salarios en México. Conclusiones. Los resultados concuerdan con la literatura internacional. Los bajos salarios, malas condiciones laborales y escasas posibilidades de desarrollarse profesionalmente en México contribuyen a generar la expectativa de migrar en la población de estudio. Adicionalmente, la percepción optimista de los estudiantes sobre el mercado extranjero y la demanda laboral de países desarrollados coadyuvan a enfatizar dicho fenómeno.

Palabras clave: estudiantes de enfermería; intención; empleo; migración internacional; México
Rosales-Martínez Y, Nigenda G, Galárraga O, Ruiz-Larios JA. Migration expectations among nursing students in Mexico City.

Salud Publica Mex 2010;52:244-253.

\section{Abstract}

Objective. To analyze the factors associated with the expectations to migrate abroad among nursing students in Mexico City. Material and Methods. A cross-sectional study was conducted with a non-random sample of 420 students. A logistic regression model was estimated. Results. A total of $69 \%$ of the informants expressed their intention to move abroad, to look for employment (65\%) and/or to continue their studies (26\%). Of those, $50 \%$ would choose Canada as their destination, followed by Spain and the United States. The variables associated with migration expectations were: age, income, having relatives abroad, and perception of poor labor conditions and low wages in Mexico. Conclusions. Results are consistent with international literature. Low wages, poor labor conditions and the limited possibilities for professional development in Mexico are factors that contribute to generate migration expectations among nursing students. Additionally, optimistic perceptions about the job market and the labor demand in more developed countries heighten expectations to migrate.

Keywords: Students, nursing; intention; employment; emigration and immigration; Mexico

(I) Centro de Investigaciones en Sistemas de Salud, Instituto Nacional de Salud Pública. Cuernavaca, Morelos, México.

Fecha de recibido: 17 de julio de 2009 - Fecha de aceptado: 4 de febrero de 2010 Solicitud de sobretiros: Dr. Gustavo Nigenda. Centro de Investigaciones en Sistemas de Salud. Instituto Nacional de Salud Pública. Av. Universidad 655, Col. Santa María Ahuacatitlán. 62100 Cuernavaca, Morelos, México. Correo electrónico: gnigenda@insp.mx 
L migración del personal de enfermería de países en desarrollo a países industrializados es un fenómeno conocido y documentado. En México no se conoce el volumen de enfermeras/os que logra migrar a otros países para trabajar en el campo de su entrenamiento. No obstante, existen indicios de que este fenómeno podría aumentar cuantitativamente de manera importante, dadas las tendencias demográficas y epidemiológicas de los países tradicionalmente receptores y el control que en ellos existe sobre la formación de personal de salud especializado. ${ }^{1}$

En el ámbito internacional, el flujo migratorio de los y las enfermeras ha respondido a la necesidad de subsanar el déficit de este personal en países industrializados. Entre los principales factores de atracción, como ocurre con otros grupos poblacionales, destacan los salarios vigentes en los países receptores. ${ }^{2}$

Lo documentado en la literatura internacional ofrece una idea de la magnitud del problema. En 2002, $85 \%$ del personal de enfermería nacido y entrenado en Filipinas se encontraba empleado en el mercado internacional. ${ }^{3}$ Por otro lado, en $200343 \%$ de los y las enfermeras que laboraba en Reino Unido había sido formado en otros países. ${ }^{4}$

En América Latina, personal de enfermería de Costa Rica, Honduras, Nicaragua, Panamá, Cuba, Puerto Rico, Argentina, Perú y Paraguay es atraído por el mercado de salud de EU, Canadá, Italia y España. Estos países estimulan dicho flujo migratorio a través de agencias de contratación internacional que ofrecen paquetes laborales atractivos para personal recién egresado de esta carrera. Los porcentajes de migración en esta región son muy variados. En 2005, se reportó que $2.3 \%$ del total de personal de enfermería argentino migró a otro país, así también personal originario de Brasil (2.8\%), Guatemala (34\%), Perú (15\%) y Panamá $(46.8 \%))^{5}$

En México, los registros sobre el tema hacen referencia a flujos migratorios de personal de enfermería desde principios de los años 90 teniendo como destinos principales Estados Unidos, España y Reino Unido. ${ }^{6}$ Otro estudio reveló que el Estado de México y Chihuahua reportaron en 2003 la migración de 150 y 500 personas con estudios de enfermería respectivamente, como respuesta a un déficit de personal en Texas, California, Florida y Nueva York.?

Entre las principales repercusiones de la migración de enfermeras y enfermeros para los países proveedores se encuentran, por un lado, la relación entre la insuficiencia de personal, el desempeño del sistema de salud y el efecto en la salud de la población ${ }^{8}$ y, por otro, la pérdida de la inversión pública efectuada en el entrenamiento de los y las enfermeras que posiblemente trabajarán en otro país.
La evidencia empírica sobre los determinantes de la decisión de migrar en este personal señala la relevancia del ingreso y los incentivos. En países pobres, la baja remuneración y la falta de congruencia entre el salario, las funciones laborales asignadas y el grado académico alcanzado constituyen un motivo de insatisfacción que coadyuva a generar la expectativa de migrar. ${ }^{9}$ Las características anteriores están presentes en el mercado laboral de salud mexicano y se entrelazan además con aspectos como el desempleo, subempleo y jornadas de trabajo prolongadas. ${ }^{10}$

Asimismo, algunos estudios realizados sobre la expectativa de migración internacional en personal de enfermería aportan cifras para dimensionar este fenómeno. En Zimbabwe, 71\% de las encuestadas declaró tener la intención de migrar en el futuro inmediato proyectando como país destino a Reino Unido (30\%), Sudáfrica $(24 \%)$ y el resto a Australia, EU, Nueva Zelanda y Canadá. ${ }^{11}$ Hallazgos similares fueron difundidos a partir de otra investigación realizada en seis países de África con diferentes categorías profesionales, de los cuales $49.8 \%$ tenía como profesión enfermería. La proporción resultante de profesionales que manifestó la expectativa de migrar se ubicó en el rango de $26 \%$ en Uganda y $68 \%$ en Zimbabwe. ${ }^{12}$

En virtud de la tendencia mundial creciente del reclutamiento de personal de enfermería, de sus repercusiones en los sistemas de salud de los países expulsores y del escaso conocimiento sobre lo que ocurre en México sobre el tema, este estudio busca conocer y analizar algunos de los factores que explican la expectativa de migración internacional de un grupo de estudiantes de enfermería en el último año de la licenciatura en tres escuelas públicas en el Distrito Federal. La importancia de conocer dicha expectativa consiste en la oportunidad de disponer de un diagnóstico sobre el fenómeno en un contexto particular e impulsar investigación al respecto a escala nacional a partir de los resultados obtenidos.

Entre sus aportaciones, este estudio enriquecerá el análisis relativo a la planeación estratégica de los trabajadores de la salud en México y contribuirá a justificar, con base en evidencia, la formulación de políticas de retención y distribución de recursos humanos en el plano regional, nacional e internacional.

\section{Material y métodos}

Se realizó un estudio transversal con una muestra no probabilística de 420 estudiantes. El trabajo de campo se llevó a cabo a mediados de 2007 en el Distrito Federal. La unidad de análisis fueron los estudiantes de licenciatura en enfermería inscritos en tres escuelas seleccionadas que estuvieran cursando el último año de la carrera y 
estuviesen presentes el día del levantamiento de los datos. El único criterio de exclusión consistió en no mostrar interés en participar. La elección de este grupo poblacional respondió a la relevancia de su condición como futuros profesionales de la salud y a la viabilidad metodológica que representó tenerlos reunidos en un espacio físico delimitado.

Se llevó a cabo una encuesta empleando un instrumento previamente probado en forma piloto con estudiantes de enfermería de la Universidad Autónoma del Estado de Morelos. El cuestionario se conformó de seis apartados que indagaron: datos sociodemográficos, desempeño académico, fuentes de información sobre migración internacional, percepción sobre condiciones del mercado laboral en México, expectativa de migrar y percepción sobre condiciones del mercado laboral internacional. Para el diseño de las preguntas se tomó como referencia los testimonios recabados en un grupo focal con estudiantes de enfermería y los resultados de una revisión bibliográfica.

Para la selección de las escuelas e informantes se utilizó el muestreo por criterio. Se buscó que las escuelas fueran de control público y que de acuerdo con el Anuario estadístico de la Asociación Nacional de Universidades e Instituciones de Educación Superior (ANUIES) registraran el mayor número de egresados de esta licenciatura en el Distrito Federal. A partir de estos criterios se eligió a la Escuela Nacional de Enfermería y Obstetricia (ENEO) de la Universidad Nacional Autónoma de México (UNAM); la División de Ciencias Biológicas y de la Salud (DCBS) de la Universidad Autónoma Metropolitana (UAM); y la Escuela Superior de Enfermería y Obstetricia (ESEO) del Instituto Politécnico Nacional (IPN), que juntas egresan anualmente alrededor de $72 \%$ de licenciados en enfermería en esta entidad.

En cuanto a los informantes, se trabajó con una muestra de sujetos voluntarios. La aplicación del cuestionario se llevó a cabo durante eventos organizados por las escuelas donde se convocaron a estudiantes de los últimos semestres para la inducción al servicio social. Únicamente en el caso de la ENEO los estudiantes fueron convocados específicamente para participar en este estudio. El instrumento fue respondido por los y las estudiantes sin intermediación de un entrevistador. En promedio, el porcentaje de participación en la encuesta con respecto a las matrículas totales de los últimos semestres de la carrera en las tres escuelas fue de 77.6\%.

En términos logísticos, se contactó a autoridades de las tres escuelas mediante oficio institucional y protocolo de investigación anexo. Se solicitó su aprobación y apoyo para el trabajo de campo. Asimismo, el proyecto fue aprobado por la Comisión de Ética del Instituto Nacional de Salud Pública. Para cumplir con las consideraciones éticas de la investigación se adjuntó a los cuestionarios una carta de consentimiento informado donde se dio a conocer a las y los estudiantes los objetivos del estudio y se garantizó la confidencialidad de sus respuestas.

\section{Análisis de los datos}

Se realizó una regresión logística multivariada para estimar la asociación entre una serie de variables independientes y una respuesta dicotómica relativa a la presencia o ausencia de la expectativa de migrar. Se compararon las características socioeconómicas y la percepción sobre las condiciones laborales en México y en el extranjero a partir de la variable dependiente utilizando la prueba ji². Finalmente, se construyó un modelo multivariado con variables seleccionadas a partir de la teoría reportada y aquellas con asociación estadísticamente significativa.

El número de observaciones en el modelo multivariado fue de 403 y la probabilidad del conjunto de variables independientes fue significativa $(p<0.000)$. Para verificar la bondad de ajuste se usó la prueba de $\mathrm{ji}^{2}$ de Hosmer-Lemeshow concluyendo con una $p>0.05$ que el modelo es útil para explicar el fenómeno estudiado en tanto las probabilidades estimadas son iguales a las probabilidades observadas. Se aplicó el diagnóstico de colinealidad de las variables con la prueba de tolerancia y factor de inflación de la varianza (VIF, por sus siglas en inglés) para verificar que las variables independientes no presentaran una alta correlación entre ellas. Para el procesamiento de los datos se usó el paquete estadístico Stata. ${ }^{*}$ A pesar de que no se encontraron diferencias por sexo, esta variable fue incluida en el modelo ajustado debido a que su presencia incrementaba la significancia estadística del resto de las variables. Adicionalmente, se construyó un índice de diferencia (ID) a partir de dos preguntas cerradas diseñadas con opciones de respuesta idénticas y, por tanto, comparables entre sí. En el cuestionario se colocaron cuatro opciones para responder a las preguntas: $¿$ Conseguir empleo en una institución de salud (en México o en el extranjero) para una enfermera recién egresada de licenciatura es? a) fácil, b) complicado, c) imposible, d) no sabe. El ID se creó restando las observaciones incluidas en cada pregunta sobre "oportunidades en México" vs. "oportunidades en el extranjero". Un valor positivo en el ID indica que la percepción de la situación de empleo en México es mejor (o más alta) que aquella en el extranjero. Por el contrario, un valor negativo del ID implica que las opor-

\footnotetext{
* StataCorp. Stata Statistical Software: Release 8. College Station TX, USA, StataCorp LP, 2005.
} 
tunidades en el extranjero se perciben como superiores (o más altas). Finalmente, un valor de cero implica que se perciben como equivalentes las oportunidades de empleo en ambos países.

\section{Resultados}

El cuestionario fue respondido por 420 estudiantes de enfermería de los cuales $85.5 \%$ fueron mujeres. El 78\% se ubicó en el rango de edad de 20 a 24 años. El principal lugar de origen reportado fue el Distrito Federal $(80 \%)$, seguido del Estado de México (10\%). La mayor parte de los informantes (82\%) declaró ser soltero(a) y sólo 16\% mencionó tener dependientes económicos. Respecto al ingreso promedio mensual en el hogar, 53\% declaró percibir hasta dos salarios mínimos, mientras que $21 \%$ reportó un ingreso de seis salarios mínimos en adelante. En cuanto a su actividad, además de dedicarse a los estudios, $23 \%$ se encontraba trabajando. De estos últimos, $73 \%$ recibía un ingreso promedio mensual que no superaba los dos salarios mínimos. Finalmente, $55 \%$ de los encuestados declaró tener familiares en el extranjero.

Se encontraron diferencias significativas $(p<0.05)$ de acuerdo con la edad. En el grupo de estudiantes entre 20 y 24 años se concentró la proporción más alta con expectativa de migrar. También se encontraron diferencias respecto a tener o no familiares en el extranjero. En el grupo de mujeres se presentó mayor proporción de la expectativa de migrar, pero la diferencia no fue significativa (cuadro I). La variable desempeño académico fue medida; sin embargo, no contribuyó a explicar el fenómeno.

En relación con la variable de interés, $69 \%(\mathrm{~N}=287)$ declaró tener la expectativa de migrar. El 65\% de los informantes reportó que migraría con la intención de ejercer su carrera y $26 \%$ para continuar estudiando. En cuanto al país destino de preferencia, 50\% elegiría Canadá, seguido de España (27\%), Estados Unidos (16\%) y otros $(7 \%)$. En la figura 1 se muestran los resultados sobre la principal razón que lleva a los licenciados en enfermería a migrar según la percepción de los encuestados. En este sentido, casi la mitad de ellos (49\%) atribuyó esta situación a los bajos salarios en México.

En cuanto a las fuentes y tipo de información sobre migración internacional de personal de enfermería, $84 \%$ manifestó disponer de información al respecto. De ellos 33\% la obtuvo por iniciativa propia. El 52\% dijo estar informado sobre condiciones de empleo en otros países y la mitad apuntó al menos un requisito para ser contratado en el extranjero. Entre estos requisitos, los más mencionados fueron conocer el idioma del país receptor, estar titulado y tener los documentos migratorios en regla.

E135\% reportó haber tenido tres diferentes fuentes de información vinculadas con condiciones laborales en otros países y $27 \%$ declaró tener hasta cuatro fuentes. En la figura 2 se pueden apreciar las fuentes de información mencionadas. Las dos principales fueron profesores $(25 \%)$ e Internet (16\%). Asimismo, 54\% señaló tener familiares viviendo fuera de México, en su mayoría tíos y primos; de ellos $12 \%$ reconoció a dichos familiares como fuente de información sobre migración internacional.

En el cuadro II se presentan algunas características vinculadas con la percepción de las condiciones laborales en México y en el extranjero. Al comparar las frecuencias de aquellos que manifestaron tener y no tener la expectativa de migrar se encontraron diferencias estadísticamente significativas. Dentro del grupo de estudiantes con la expectativa de migrar se registró una proporción mayor de estudiantes que percibían mayor oportunidad de conseguir empleo en el extranjero. Asimismo, en el grupo que calificó como malas las condiciones laborales y los salarios en México fue mayor el porcentaje de estudiantes con la expectativa de migrar. Por último, entre aquellos que percibían a Canadá y España como los mejores países de destino y que calificó como mejores los salarios para personal de enfermería en el extranjero también se presentó una mayor proporción de estudiantes con dicha intención.

En el cuadro III se muestran las variables ajustadas que se asociaron significativamente con la expectativa de migrar. Se estimó que la posibilidad de tener esta expectativa se presenta en estudiantes que pertenecen al grupo de edad más joven (20-24 años), con un ingreso promedio en el hogar superior a los cinco salarios mínimos y con familiares viviendo en otros países. Asimismo, esta posibilidad aumenta cuando los estudiantes perciben malas condiciones laborales en México aunado a considerar mejores los salarios ofertados a personal de enfermería en el extranjero.

\section{Discusión}

En general, los resultados obtenidos sobre los determinantes de la expectativa de migrar encontraron correspondencia con la literatura internacional. Entre los factores sociodemográficos asociados con este fenómeno se encontró la edad ${ }^{13}$ y el ingreso en el hogar. Se observó una tendencia generacional a presentar mayor intención de migrar en los grupos de informantes más jóvenes. Esto sugiere que la edad es un factor de decremento de la expectativa de migrar dado que los jóvenes tienen menor aversión al riesgo en comparación con la 
Cuadro I

Características sociodemográficas de los estudiantes Según LA EXPECTATIVA de Migrar.

ENCUESTA A ESTUDIANTES DE LA LICENCIATURA EN ENFERMERÍA EN TRES ESCUELAS PÚBLICAS.

Distrito Federal, MAYO Y JUNIO de 2007

Variables

\begin{tabular}{|c|c|c|c|c|c|}
\hline \multirow{3}{*}{ Variables } & & \multirow{3}{*}{$p\left(j i^{2}\right)$} \\
\hline & \multicolumn{2}{|c|}{ No } & \multicolumn{2}{|c|}{ Si } & \\
\hline & $N=|3|$ & $\%$ & $N=287$ & $\%$ & \\
\hline \multicolumn{6}{|l|}{ Sexo } \\
\hline Hombre & 21 & 16.03 & 39 & 13.59 & \\
\hline Mujer & 110 & 83.97 & 248 & 86.41 & 0.509 \\
\hline \multicolumn{6}{|l|}{ Grupo de edad } \\
\hline $20-24$ & 91 & 69.47 & 235 & 82.46 & \\
\hline $25-46$ & 40 & 30.97 & 50 & 17.54 & 0.003 \\
\hline \multicolumn{6}{|l|}{ Lugar de origen } \\
\hline Distrito federal & 113 & 86.26 & 225 & 78.40 & \\
\hline Otra entidad federativa & 18 & 13.74 & 62 & 21.60 & 0.058 \\
\hline \multicolumn{6}{|l|}{ Estado civil } \\
\hline Soltero & 108 & 82.44 & 233 & 81.18 & \\
\hline Casado/unión libre & 21 & 16.03 & 46 & 16.03 & \\
\hline Separado/divorciado & 2 & 1.53 & 8 & 2.79 & 0.735 \\
\hline \multicolumn{6}{|l|}{ Escolaridad del padre } \\
\hline Ninguna & 4 & 3.10 & 12 & 4.26 & \\
\hline Primaria & 34 & 26.36 & 77 & 27.30 & \\
\hline Secundaria & 43 & 33.33 & 81 & 28.72 & \\
\hline Carrera técnica o comercial & 13 & 10.08 & 26 & 9.22 & \\
\hline Preparatoria o vocacional & 17 & 13.18 & 46 & $|6.3|$ & \\
\hline Licenciatura & 12 & 9.30 & 31 & 10.99 & \\
\hline Maestría & 2 & 1.55 & 2 & 0.71 & \\
\hline No sabe & 4 & 3.10 & 7 & 2.48 & 0.919 \\
\hline \multicolumn{6}{|l|}{ Ingreso promedio hogar (pesos) } \\
\hline Menos de 1,400 & 16 & $|2.2|$ & 27 & 9.57 & \\
\hline $1,400-4,000$ & 59 & 45.04 & 116 & 41.13 & \\
\hline $4,001-6,700$ & 38 & 29.01 & 70 & 24.82 & \\
\hline Más de 6,700 & 18 & 13.74 & 69 & 24.47 & 0.093 \\
\hline \multicolumn{6}{|l|}{ Dependientes económicos } \\
\hline No & 113 & 86.26 & 239 & 83.28 & \\
\hline Sí & 18 & 13.74 & 48 & 16.72 & 0.438 \\
\hline \multicolumn{6}{|l|}{ Empleo } \\
\hline No & 106 & 82.81 & 212 & 74.91 & \\
\hline Sí & 22 & 17.19 & $7 \mid$ & 25.09 & 0.076 \\
\hline \multicolumn{6}{|l|}{ Ingreso empleo (pesos) } \\
\hline Menos de I,400 & 6 & 27.27 & 20 & 28.17 & \\
\hline$I, 40 \mid-4,000$ & 14 & 63.63 & 27 & 38.03 & \\
\hline Más de 4,000 & 2 & 9.09 & 23 & 31.39 & 0.054 \\
\hline \multicolumn{6}{|l|}{ Familiares en el extranjero } \\
\hline No & 74 & 56.49 & 115 & 40.35 & \\
\hline Sí & 57 & 43.51 & 170 & 59.65 & 0.002 \\
\hline
\end{tabular}

Expectativo 


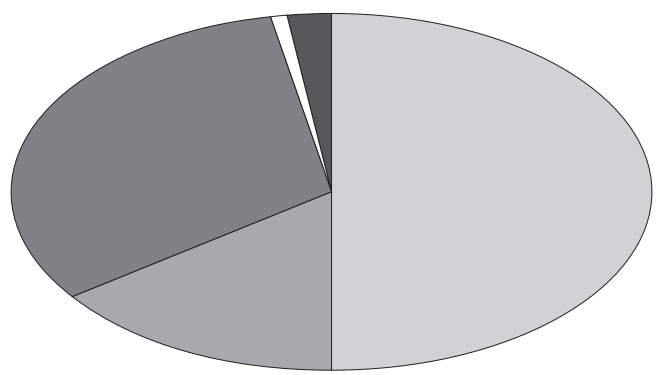

Los bajos salarios en México

La dificultad para conseguir empleo

Que no se valora a los licenciados en enfermería

Que tienen familiares viviendo en ese país

Otro

Figura I. Principales razones Que llevan a las enferMERAS A MIGRAR. ENCUESTA A ESTUDIANTES DE LA LICENCIATURA EN ENFERMERÍA EN TRES ESCUELAS PÚBLICAS. DISTRITO Federal, MAYO Y JUNIO dE 2007

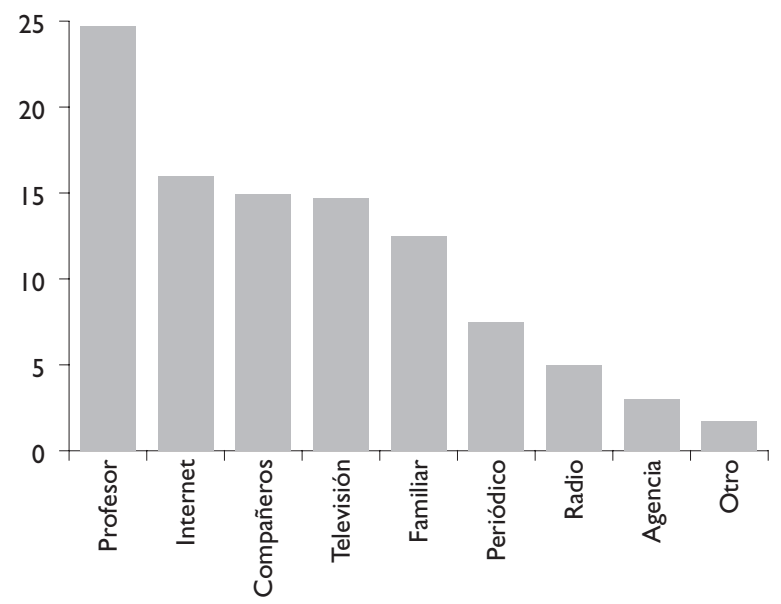

Fuente: Encuesta a estudiantes de la licenciatura en enfermería en tres escuelas públicas del Distrito Federal durante mayo y junio de 2007

Figura 2. Fuentes de iNFormaCión SOBRE MIGRACIÓN INTERNACIONAL DE ENFERMERAS. ENCUESTA A ESTUDIANTES DE LA LICENCIATURA EN ENFERMERÍA EN TRES ESCUELAS PÚBLICAS. Distrito Federal, MAYO Y JUNIO de 2007

población adulta. ${ }^{14}$ Respecto al ingreso en el hogar, se observó que la estratificación socioeconómica al interior del gremio perfila diferentes aspiraciones en términos educativos y/o laborales, lo que se expresa en la búsque- da de mejores condiciones de empleo, en este caso fuera de México. Mientras más alto se encuentre la familia en la escala socioeconómica, más clara es esta preferencia por parte de los estudiantes.

Una posible explicación sobre este fenómeno surge a partir de las mejores alternativas que ofrecen los mercados profesionales en términos de ingresos y beneficios a diferencia de los mercados no especializados donde la mayoría de los migrantes mexicanos busca ubicarse. El personal de enfermería planifica la migración tras evaluar las alternativas de empleo tanto en su país como en el extranjero. Esta evaluación implica dos factores importantes. Por un lado, el estatus profesional logrado después de obtener un grado universitario y, por otro, la demanda específica de enfermeras/os por parte de países desarrollados. Estos aspectos se intensifican en estudiantes cuyas familias poseen mayores ingresos ya que migrar sólo tendrá sentido si pueden mantener o superar el estatus de vida logrado en su país de origen. ${ }^{15}$

Lo anterior puede ser reforzado a partir de la correlación entre el ingreso socioeconómico de la familia y las oportunidades de acceso al sistema educativo identificado por Fernández. ${ }^{16}$ Este autor señala que a medida que se avanza en los grados y niveles del sistema educativo, éste tiende a hacerse más discriminatorio para los alumnos cuyas familias pertenecen a estratos sociales más bajos. Nuestro estudio muestra que incluso al interior de las escuelas profesionales, el nivel de ingreso familiar juega un papel en el desarrollo de la expectativa de migrar no como una medida desesperada, sino como un proceso planificado de inversión en capital humano que permita encontrar un nicho laboral de alta renta, o continuar con la formación especializada, incluso en el mercado internacional. En este sentido, cabe resaltar que 26\% de los informantes con la expectativa de migrar señalaron como principal motivo continuar estudiando en otro país ya que percibían menos prometedora esta oportunidad en su país de origen.

Un factor fuertemente asociado con la expectativa de migrar es la expectativa del salario y las condiciones laborales del mercado internacional. Si bien la literatura menciona en primera instancia las razones económicas en este fenómeno, también provee evidencia de la influencia de otros aspectos. Algunos autores señalan la insatisfacción en la vida profesional como un predictor poderoso en la planeación de migrar al extranjero. ${ }^{17,18}$ En general, además de un salario atractivo se buscan mejores condiciones de contratación e incentivos no financieros así como oportunidades de desarrollo profesional, gestión en el trabajo, entornos laborales positivos y acceso a prestaciones. ${ }^{19}$

El mercado laboral de enfermería en México ofrece condiciones que motivan a las y los egresados universitarios a migrar. México se ha distinguido por ofrecer 
Cuadro II

Percepción de condiciones laborales en México y en el extranjero, Según la expectativa de migrar.

ENCUESTA A ESTUDIANTES DE LA LICENCIATURA EN ENFERMERÍA EN TRES ESCUELAS PÚBLICAS.

Distrito Federal, MAYO Y JUNIO de 2007

Variables

Oportunidad de conseguir empleo México - extranjero*

Más fácil en México

No percibe ninguna diferencia

Más fácil en el extranjero

No sabe

\begin{tabular}{ccccc}
\multicolumn{3}{c}{ Expectativa } \\
\cline { 1 - 1 } & No & Si $\left(\mathrm{ji}^{2}\right)$
\end{tabular}

$\begin{array}{rr}3 & 2.29 \\ 55 & 41.98 \\ 32 & 24.43 \\ 41 & 31.30\end{array}$

$\begin{array}{rr}8 & 2.81 \\ 129 & 45.26 \\ 103 & 36.14 \\ 45 & 15.79\end{array}$

0.002

Percepción condiciones laborales en México

Muy buenas y buenas

Regulares

Muy malas y malas

No sabe

$\begin{array}{rr}36 & 27.69 \\ 72 & 55.38 \\ 21 & 16.15 \\ 1 & 0.77\end{array}$

16.03

50.87

33.10

Percepción salarios en México

Muy buenos / buenos
Regulares
Muy malos / malos
No sabe

$19 \quad 14.50$

14.50

61.83

22.14

$2 \quad 1.53$

$\begin{array}{rr}46 & 16.03 \\ 146 & 50.87 \\ 95 & 33.10 \\ 0 & 0\end{array}$

Subir en escalafón laboral (México)

Fácil

Complicado

Imposible

No sabe

29

$\begin{array}{rr}26 & 9.09 \\ 134 & 46.85 \\ 122 & 42.66 \\ 4 & 1.40\end{array}$

0.001

Posibilidad de recibir cursos de actualización en México

$\begin{array}{lrr}\text { Muy alta } & 9 & 6.87 \\ \text { Alta } & 66 & 50.3 \\ \text { Baja } & 38 & 29.0 \\ \text { Muy baja } & 7 & 5.34 \\ \text { No sabe } & 11 & 8.40\end{array}$

10.69

$103 \quad 78.63$

I $\quad 0.76$

$13 \quad 9.92$

$\begin{array}{rr}18 & 6.29 \\ 247 & 86.36 \\ 6 & 2.10 \\ 15 & 5.24\end{array}$

0.079

Pís con mejores condiciones laborales

$\begin{array}{lrr}\text { España } & 15 & 11.63 \\ \text { Canadá } & 64 & 49.6 \\ \text { Estados Unidos } & 41 & 31.78 \\ \text { Otro } & 2 & 1.55 \\ \text { No sabe } & 7 & 5.43\end{array}$

Salarios en otros países en comparación con México

Mejores

Iguales

Peores

No sabe

$\begin{array}{rr}100 & 76.92 \\ 2 & 1.54 \\ 0 & 0 \\ 28 & 21.54\end{array}$

$\begin{array}{rr}259 & 91.20 \\ 8 & 2.82 \\ 0 & 0 \\ 17 & 5.99\end{array}$

0.878

*Variable creada a partir de un índice de diferencia 


\section{Cuadro III}

ANÁlisis MUlTIVARIADO DE REGRESIÓN LOGísticA EN ESTUDIANTES CON LA EXPECTATIVA DE MIGRAR AL EXTRANJERO.

ENCUESTA A ESTUDIANTES DE LA LICENCIATURA EN ENFERMERÍA EN TRES ESCUELAS PÚBLICAS.

Distrito Federal, MAYO Y JUNIO de 2007

\begin{tabular}{|c|c|c|c|}
\hline \multirow{2}{*}{ Variables } & \multicolumn{3}{|c|}{ Modelo multivariado } \\
\hline & $\begin{array}{c}R M \\
\text { ajustada }\end{array}$ & (IC 95\%) & $p$ \\
\hline \multicolumn{4}{|l|}{ Edad } \\
\hline $\begin{array}{r}20-24 \\
25-46\end{array}$ & $\begin{aligned} 2.02 \\
I^{*}\end{aligned}$ & $1.18-3.46$ & 0.009 \\
\hline \multicolumn{4}{|l|}{ Sexo } \\
\hline Hombre & I* & & \\
\hline Mujer & 1.37 & $.726-2.61$ & 0.325 \\
\hline \multicolumn{4}{|l|}{ Ingreso hogar / (SMM) (pesos) } \\
\hline Hasta I,400 (I) & $I^{*}$ & & \\
\hline $1,500-4,000(>1-3)$ & 1.17 & $.55 I-2.50$ & 0.674 \\
\hline $4,100-6,700(>3-5)$ & 1.12 & $.505-2.51$ & 0.767 \\
\hline Más de 6,800 (>5) & 2.59 & $1.06-6.32$ & 0.036 \\
\hline \multicolumn{4}{|l|}{ Familiares en el extranjero } \\
\hline No & I* & & \\
\hline Sí & 1.75 & $1.11-2.76$ & 0.015 \\
\hline \multicolumn{4}{|l|}{ Condiciones laborales en México } \\
\hline Muy buenas/buenas & $I^{*}$ & & \\
\hline Regulares & 1.48 & $.845-2.59$ & 0.169 \\
\hline Malas/muy malas & 3.33 & $1.67-6.62$ & 0.001 \\
\hline \multicolumn{4}{|l|}{ Salarios en el extranjero vs. México } \\
\hline Mejores & 3.63 & $1.84-7.17$ & \\
\hline Iguales & 4.66 & $0.78-27.66$ & 0.000 \\
\hline No sabe & $I^{*}$ & & 0.090 \\
\hline \multicolumn{4}{|l|}{ * Referencia } \\
\hline \multicolumn{4}{|c|}{$\begin{array}{l}\text { Numero de observaciones }=403 ; \text { Hosmer-Lemeshow } \mathrm{ji}^{2}(8)=3.23 ; \text { Prob }>\mathrm{ji}^{2}=0.9191 \\
\text { SMM: salario mínimo mensual }\end{array}$} \\
\hline
\end{tabular}

condiciones laborales precarias a los profesionales en salud que no han mejorado con las reformas del sector impulsadas a partir de los años ochenta. Una de las principales tendencias del mercado laboral ha sido la sustitución de relaciones de trabajo estables y protegidas por esquemas flexibles de contratación y sistemas de incentivos basados en estrategias de contención de costos..$^{20}$ En este contexto, los profesionales de la salud enfrentan un futuro laboral incierto ante la supresión de beneficios sociales, el deterioro de los salarios y una débil regulación de sus derechos como trabajadores por parte del Estado. ${ }^{21}$

Complementariamente, las diferencias económicas entre regiones, la escasez de personal de enfermería en países desarrollados y la consecuente demanda interna- cional del mismo agudizan la expectativa de migrar. ${ }^{22} \mathrm{De}$ esta forma, la globalización de los mercados de trabajo se convierte en una opción de supervivencia que transfiere a otras regiones las demandas laborales y profesionales no atendidas en los países de origen.

Desde un enfoque cultural, estos procesos sociales y económicos son interiorizados por los sujetos generando en ellos un conjunto de expectativas sobre las oportunidades de desempeñarse en otros países. ${ }^{23}$ Ello desemboca gradualmente en una aceptación colectiva del fenómeno. En este escenario, la posibilidad de ejecutar políticas adecuadas para regular el proceso de migración de trabajadores de la salud se vuelve urgente.

El estudio de las expectativas de migración entre enfermeras y enfermeros mexicanos permite contar con 
un primer indicador sobre la posibilidad de que este fenómeno aumente en el país. Hasta la fecha, la migración ha mantenido una proporción relativamente baja para el personal de enfermería mexicano como opción laboral. Sin embargo, los déficits pronosticados de este personal en los EUA y otros países industrializados, aunados a los procesos de envejecimiento de su población, generan un escenario de alta probabilidad migratoria en los próximos años. A esto se deben sumar las poco atractivas condiciones laborales existentes en México. En términos de su práctica profesional, el personal de enfermería constituye un recurso humano importante en razón de la cobertura de atención que brindan y su consecuente contribución en el mejoramiento de las condiciones de salud de las poblaciones. Se ha documentado que el mejoramiento del cuidado obstétrico y la disminución de la mortalidad materna son adjudicados en gran medida al desempeño de este personal. ${ }^{24}$

En el supuesto de acentuarse esta migración, México enfrentaría impactos de índole social y económica. Uno de ellos sería la pérdida de la inversión pública realizada en el entrenamiento de este personal. ${ }^{25}$ Otro más sería el efecto negativo en la salud de la población debido a la insuficiencia de personal y el bajo desempeño de las instituciones de salud. ${ }^{26}$ Las metodologías empleadas para evaluar el desempeño de los sistemas de salud consideran el número de recursos humanos como un indicador de la capacidad de atención. ${ }^{27}$ Según la OCDE, la disponibilidad de personal de enfermería en México es de 2.2 por cada mil habitantes, mientras que el promedio de los países que integran esta organización es de 8.0 por cada mil. ${ }^{28}$

En virtud de que el volumen de personal de enfermería migrante es todavía bajo, este es un momento para que las autoridades competentes diseñen y pongan en práctica una política que regule el eventual crecimiento migratorio a fin de garantizar el respeto a los derechos laborales y humanos a las personas que decidan tomar esta opción y que proteja a las instituciones de salud nacionales en el caso que tengan que prescindir de un recurso tan valioso.

En la lógica de regular la migración, las estrategias en el terreno laboral pueden ir encaminadas a la ampliación de los sistemas de incentivos financieros y no financieros, de tal forma que beneficien a todo el personal con licenciatura en enfermería independientemente de la institución pública empleadora y el esquema de contratación.

Otra estrategia podría estar enfocada a difundir información fidedigna por parte de fuentes oficiales para equilibrar el efecto de fuentes de información sobre migración internacional en los estudiantes. Asumiendo la influencia de éstas, valdría la pena reorientar los men- sajes en medios de comunicación para brindar información fidedigna acerca de las condiciones laborales para el personal de enfermería en el extranjero. Asimismo, el uso de sistemas de información ya existentes constituye un recurso que puede ser complementado con indicadores que permitan conocer el número de enfermeras y enfermeros que migran por la vía autorizada, así como el tiempo que permanecen en otro país.

Queda pendiente para futuras investigaciones analizar la presencia y funcionamiento de las agencias de reclutamiento internacional en México como instancias que promueven el proceso migratorio. Asimismo, sería recomendable en el marco de la demanda internacional de personal de enfermería fomentar investigaciones interesadas en estudiar el impacto que tendrían las instituciones de salud en México si la migración de este personal se masifica, así como analizar sus condiciones laborales como un mecanismo que podría ayudar a regular el mercado.

\section{Agradecimientos}

Este estudio se realizó con recursos del Fondo Sectorial CONACYT-SALUD por medio del proyecto "Empleo, formación y regulación de recursos humanos para la salud. Bases para su planeación estratégica". También agradecemos al equipo de trabajo en recursos humanos en salud del INSP que contribuyó en diversos aspectos en la realización del estudio.

\section{Referencias}

I.Thupayagale-Tshweneagae G. Migration of nurses: is there any other option? International Nursing Review 2007;54:107-109.

2. Organización Mundial para la Salud. Informe sobre la salud en el mundo. Colaboremos por la salud. Estrategias nacionales de desarrollo del personal sanitario. Ginebra: OMS, 2006.

3. Buchan J, Parkin T, Sochalski J. Internacional nurse mobility. Trends and policy implications. WHO/ICN 2003;5:3-40.

4. Internacional de Servicios Públicos. Hoja informativa sobre las migraciones. Foro de la ISP sobre igualdad de género [monografía en internet]. Ginebra, Suiza: ISP, 2005 [consultado 2006 agosto 28] Disponible en: http://www.psi-ca.org/.

5. Malvárez S, Castrillón M. Overview of the nursing workforce in Latin America. Serie Human Resources Development [monografía en internet]. Washington: Pan American Health Organization, 2005 [consultado 2006 abril 25]. Disponible en: http://www.icn.ch/global/Issue6LatinAmerica.pdf. 6. Nigenda G, González-Robledo LM. Trade and health between Mexico and the U.S. Mexican Health Foundation 2005;7:25

7.Arroyo-de Cordero G, Jiménez-Sánchez J. Repercusiones de la escasez versus migración de enfermeras como fenómeno global. Rev Enferm IMSS 2005; I3(I):33-40

8. Organización Mundial para la Salud. Informe mundial sobre la salud. Perfil mundial de los trabajadores sanitarios. Ginebra: OMS, 2006. 9. Organización Mundial de la Salud. Migración internacional de personal sanitario: un reto para los sistemas de salud de los países en desarrollo 
[monografía en internet]. Ginebra, Suiza: OMS, 2006 [consultado 2006 agosto 28]. Disponible en: http://apps.who.int/gb/ebwha/pdf_files/WHA59/ A59_I8-sp.pdf.

10. Nigenda G, Ruiz JA, Bejarano R, Rosales Y. Enfermeras con licenciatura en México: estimación de los niveles de deserción escolar y desperdicio laboral. Salud Publica Mex 2006;48(I):26.

III. Chicanda A. Nurse migration from Zimbabwe: analysis of recent trends and impacts. Nursing Inquiry 2005; I2(3):162-174.

12. Awases M, Gbary A, Njoni J. Migration of health professionals in six countries: a synthesis report. World Health Organization 2004;65:38-42.

13. Daniel P, Chamberlain A, Gordon F. Expectations and experiences of newly recruited Filipino nurses. British Journal of Nursing 200I; 10(4):254-265.

14. Joyce M. Richmond Cooper. Migration and market wage risk. Journal of Regional Science 1994;34(4):563-582

15.Valle $\mathrm{F}$. El egreso profesional y el empleo en la crisis. Algunos planteamientos, En: Pacheco M, Barriga D. La profesión. Su condición social e institucional. México: CESU/UNAM, 1997:138.

16. Fernández V. El encuentro con la enfermería, En: Lartigue T, Fernández V, coords. Enfermería: una profesión de alto riesgo. México: Plaza y Valdés / Universidad Iberoamericana, 1998:39.

17.Vanasse A, Scott S, Courteau J. Canadian family physicians' intentions to migrate. Associated factors. Canadian Family Physician 2009;55:396-403. 18. Organización Mundial de la Salud. Migración internacional de personal sanitario: un reto para los sistemas de salud de los países en desarrollo [monografía en internet]. Ginebra, Suiza: OMS, 2006 [consultado 2006 agosto 28]. Disponible en: http://apps.who.int/gb/ebwha/pdf_files/WHA59/ A59_18-sp.pdf.

19. Consejo Internacional de Enfermeras. Directrices: incentivos para los profesionales de atención de salud [monografía en internet]. Ginebra:OMS, 2008. [consultado 2009 noviembre]. Disponible en: http://www.who.int/management/resources/staff/en/index4.html.
20. Dal Poz M,Adams O. La reforma del sector salud y sus implicaciones para los recursos humanos: una visión panorámica. En: Cuevas L, Brito $\mathrm{P}$, coords. Presente y futuro en la formación, práctica y regulación profesional en ciencias de la salud. México:OPS/OMS, 2002:36-38. 21. Malvárez S, Castrillón M. Overview of the nursing workforce in Latin America. Serie Human Resources Development [monografía en internet]. Washington: Pan American Health Organization, 2005 [consultado 2006 abril 25]. Disponible en: http://www.icn.ch/global//ssue6LatinAmerica.pdf. 22. Blanco MC. Las migraciones contemporáneas. $2^{2}$ Edición. Madrid: Alianza, 2000:57-89.

23. González-Ruiz M. Migraciones y teoría social.Algunas consideraciones [monografía en internet]. España: Rev. Laberinto, 200I. [consultado 2008 mayo]. Disponible en: http://laberinto.uma.es/.

24. Ruiz-Rodriguez M,Wirtz V, Nigenda G. Organizational elements of health services related to a reduction in maternal mortality: the cases of Chile and Colombia. Health Policy 2009;90(2): I49-I55.

25. Didou-Aupetit S. ¿Fuga de Cerebros o Diásporas? Inmigración y emigración de personal altamente calificado en México. Revista de la Educación Superior [serie en internet] 2004 ;32(I32): [aprox. 9 Pp.]. [Consultado 2008 mayo]. Disponible en: http://www.anuies.mx/ servicios/P_anuies/publicaciones/revsup//32/0 la.html.

26. Organización Mundial para la Salud. Perfil mundial de los trabajadores sanitarios. Informe mundial sobre la salud, [monografía en internet]. Ginebra:OMS, 2006 [consultado 2008 junio]. Disponible en: http://www. who.int/whr/es/index.html.

27. Organización Panamericana de la Salud. Estrategia de cooperación con México de la OPS/OMS para el periodo 2005-2009. Calidad de los servicios y desempeño del sistema de salud. [monografía en internet]. Ginebra:OPS/OMS, 2005. [consultado 2007 enero]. Disponible en: http:// www.mex.ops-oms.org/documentos/coperacion.pdf. 28. Organización para la Cooperación y el Desarrollo Económico. Estudios de la OCDE sobre los Sistemas de Salud. México:OCDE/SSA, 2005. 